\title{
The Effect of Testosterone Replacement on Endothelial Dysfunction, Inflammation and Insulin Resistance in Male Hypogonadotrophic Hypogonadism
}

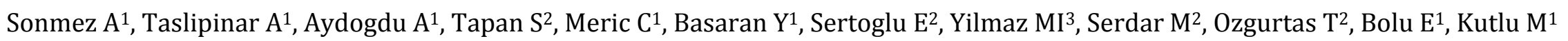 \\ Gulhane School of Medicine, Departments of ${ }^{1}$ Endocrinology, ${ }^{2}$ Biochemistry and ${ }^{3}$ Nephrology, Ankara, TURKEY
}

\section{Introduction}

Patients with hypogonadism not only present with poor libido, loss of energy, muscle atrophy and depression but also with chronic metabolic disorders such as dyslipidemia, obesity, hypertension and T2DM. The dysmetabolic features of hypogonadism are present even in the very young age, in patients with CHH. Whether TRT improves the metabolic and cardiovascular risks in hypogonadism is not clear. Several reports mention favorable metabolic effects of TRT while other studies do not confirm these data. For this purpose, we measured the markers for endothelial dysfunction, inflammation and insulin resistance as predictors for the cardiovascular mortality and morbidity. We also compared the effects of two different TRT modalities on the above parameters; triweekly injection of testosterone esters vs. daily transdermal testosterone gel.

\section{Material and Methods}

This prospective study was performed during 2008 and 2011 in the outpatient units of Gulhane Medical School. Treatment naive young patients with congenital hypogonadotrophic hypogonadism $(\mathrm{CHH})$ were enrolled $(\mathrm{n}=80$, mean age $21.56 \pm 2.1 \mathrm{yrs})$. The diagnosis of $\mathrm{CHH}$ was based on a failure to undergo spontaneous puberty before 18 years of age and was confirmed by low serum total testosterone levels, normal or low gonadotropin levels. Additional criteria were the absence of a pituitary or hypothalamic mass lesion on magnetic resonance imaging, presence of a gonadotropin response to repetitive doses of gonadotropin-releasing hormone, and a normal karyotype (46, XY). Either testosterone esters (250 mg / 3weeks IM; n=59) or testosterone transdermal gel (50 mg daily; $\mathrm{n}=21$ ) were administered in a mean follow-up period of $6.0 \pm 2.4$ months. The demographic parameters, fasting glucose, insulin, pentraxin 3 (PTX3) and asymmetric dymethyl arginine (ADMA) levels were measured both before and after treatment periods. The insulin sensitivity was estimated by HOMA-IR formula. 


\section{Results}

BMI and WC were significantly increased ( $\mathrm{p}<0.001$ and $\mathrm{p}=0.001$ respectively) and total cholesterol and HDL cholesterol levels were significantly decreased during the follow-up period. There were no significant alterations in the arterial blood pressures, Triglycerides, LDL cholesterol, fasting glucose, fasting insulin and HOMA-IR levels. There were significant increases in the ADMA and PTX-3 levels in the end of follow-up period ( $p<0.001$ and $p=0.02$ respectively). No significant difference was present between the amounts of alterations in two different treatment arms (Table-1). According to the univariate analysis, plasma ADMA levels were negatively correlated to the total testosterone levels $(\mathrm{p}=0.001, \mathrm{r}=-0.322)$. In the multiple logistic regression analysis, when the total cholesterol, PTX-3, insulin, HOMA and WC were set in the model, total testosterone level was the significant predictor of the plasma ADMA ( $\beta=-$ $0.379, \mathrm{p}=0.007$ ) (Table-2). No significant relationship was present between the alterations of the metabolic parameters and the ADMA, insulin or PTX-3 levels.

\section{Discussion}

So far, no randomized, prospective and controlled study has been performed to investigate the long-term metabolic and cardiovascular outcomes of TRT in patients with hypogonadism. Therefore, the results of the present study are important as they show the effect of TRT on the surrogate markers of cardiovascular risk, ADMA, PTX-3 and HOMA-IR levels. ADMA is the significant predictor of endothelial dysfunction, the initial step in atherosclerosis and cardiovascular mortality. PTX-3 is a novel marker of inflammation, which belongs to the same pentraxin superfamily of acute-phase reactants as C-reactive protein (CRP). However, unlike CRP, it is produced from various sources other than the liver, including the mononuclear cells and the endothelium. HOMA-IR is a reliable clinical measure of insulin resistance, which significantly predicts future risk of cardiovascular disease. The data about the effects of TRT on these parameters are not conclusive. According to our results, low testosterone levels are the significant determinants of the ADMA elevation in young and treatment naïve subjects with CHH. However, six months of TRT does not improve but worsen the ADMA, along with an increase in PTX3 levels. The results also show increased BMI and WC and decreased total and HDL cholesterol levels after TRT in these patients. The overall data may support the previous reports mentioning unfavorable cardiometabolic effects of TRT and implicate that the worsening of inflammation and endothelial dysfunction may play role in the adverse cardiovascular outcomes of TRT. The present study is also the first one to show that the cardiometabolic effects of daily transdermal and triweekly depot injections of TRT are not different. Showing the unfavorable effects of TRT on endothelial function and inflammation is utmost important due to the young age and long life expectancy of these patients. The cohort is in progress, and the data about the long-term metabolic and cardiovascular outcome of these patients will be published. 
Table-1: The effect of testosteron replacement therapy on the metabolic parameters, insulin resistance, inflammation and endothelial dysfunction.

\begin{tabular}{|c|c|c|c|c|c|c|c|c|c|}
\hline & \multicolumn{3}{|c|}{ All patients $(n=80)$} & \multicolumn{3}{|c|}{$\begin{array}{l}\text { Testosterone injection } \\
250 \mathrm{mg} / \text { 3weeks }(n=59)\end{array}$} & \multicolumn{3}{|c|}{$\begin{array}{l}\text { Testosterone transdermal gel } \\
50 \mathrm{mg} / \text { day }(\mathrm{n}=21)\end{array}$} \\
\hline & Before & After & $\mathbf{P}$ & Before & After & $\mathbf{p}$ & Before & After & $\mathbf{P}$ \\
\hline Age (years) & $21.56 \pm 2.1$ & & & $21.69 \pm 2.3$ & & & $21.20 \pm 1.7$ & & \\
\hline BMI $(\mathrm{kg} / \mathrm{cm} 2)$ & $22.39 \pm 3.1$ & $24.13 \pm 3.2$ & $<0.001$ & $22.62 \pm 3.2$ & $24.37 \pm 3.1$ & $<0.001$ & $21.84 \pm 2.8$ & $23.57 \pm 3.2$ & $<0.001$ \\
\hline $\mathrm{WC}(\mathrm{cm})$ & $85.12 \pm 10.4$ & $87.7 \pm 9.9$ & 0.001 & $86.35 \pm 10.46$ & $88.74 \pm 9.3$ & 0.024 & $82.36 \pm 10.5$ & $85.36 \pm 10.9$ & 0.006 \\
\hline SBP(mmHg) & $117.96 \pm 13.0$ & $120.04 \pm 11.3$ & 0.272 & $114.9 \pm 9.9$ & $118.65 \pm 10.8$ & 0.138 & $123.22 \pm 15.8$ & $122.44 \pm 11.9$ & 0.784 \\
\hline DBP(mmHg) & $72.3 \pm 7.7$ & $71.4 \pm 9.4$ & 0.567 & $72.29 \pm 7.7$ & $71.84 \pm 9.6$ & 0.849 & $72.39 \pm 8.2$ & $70.5 \pm 9.3$ & 0.425 \\
\hline FBG (mg/ dl) & $86.4 \pm 7.3$ & $86.8 \pm 7.9$ & 0.723 & $85.96 \pm 7.5$ & $88.04 \pm 7.6$ & 0.133 & $87.78 \pm 6.44$ & $83.47 \pm 8.1$ & 0.08 \\
\hline TG (mg/ dl) & $109.8 \pm 61.6$ & $119.2 \pm 73.0$ & 0.187 & $105.52 \pm 48.5$ & $118.26 \pm 74.2$ & 0.158 & $121.94 \pm 89.6$ & $121.78 \pm 71.5$ & 0.987 \\
\hline T. Chol. (mg/ dl) & $163.5 \pm 26.8$ & $154.0 \pm 28.2$ & 0.002 & $163.04 \pm 27.8$ & $152.79 \pm 30.24$ & 0.006 & $165.06 \pm 23.9$ & $158.18 \pm 20.3$ & 0.173 \\
\hline HDL Chol. (mg/ dl) & $47.6 \pm 10.0$ & $41.5 \pm 9.3$ & $<0.001$ & $48.22 \pm 10.72$ & $41.27 \pm 9.3$ & $<0.001$ & $45.94 \pm 7.37$ & $42.1 \pm 9.46$ & 0.01 \\
\hline LDL Chol. (mg/ dl) & $93.1 \pm 21.1$ & $89.9 \pm 24.1$ & 0.188 & $93.66 \pm 21.5$ & $91.4 \pm 24.2$ & 0.449 & $91.51 \pm 20.2$ & $85.58 \pm 23.6$ & 0.153 \\
\hline Hb (mg/ dl) & $13.7 \pm 0.9$ & $14.0 \pm 1.1$ & 0.05 & $13.6 \pm 1.0$ & $14.1 \pm 1.2$ & 0.04 & $13.9 \pm 0.8$ & $14.0 \pm 0.7$ & 1.0 \\
\hline Het (mg/ dl) & $40.54 \pm 2.8$ & $41.8 \pm 3.1$ & 0.005 & $40.4 \pm 3.1$ & $42.0 \pm 3.5$ & 0.04 & $41.0 \pm 2.1$ & $41.2 \pm 1.6$ & 0.7 \\
\hline Insulin $(\mu \mathrm{IU} / \mathrm{ml})$ & $9.45 \pm 4.0$ & $9.83 \pm 6.6$ & 0.46 & $9.86 \pm 4.0$ & $9.78 \pm 4.0$ & 0.899 & $8.29 \pm 4.05$ & $9.96 \pm 6.34$ & 0.125 \\
\hline
\end{tabular}




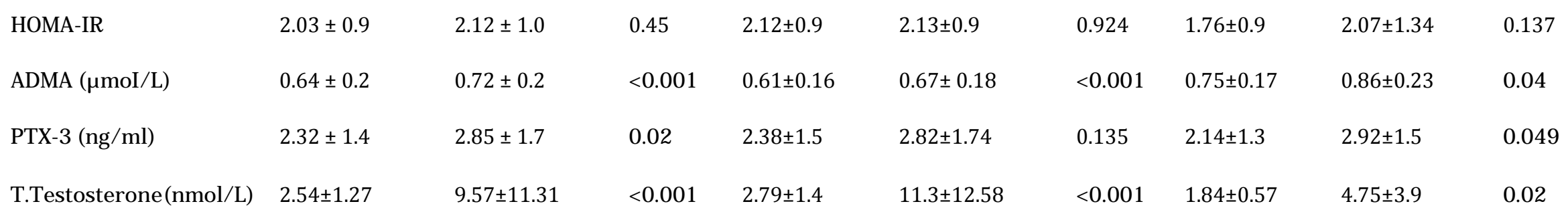

Table-2: The determinants of plasma ADMA levels in the multiple logistic regression analysis.

\begin{tabular}{lll} 
& Beta & $\mathbf{P}$ \\
\hline Total testosterone & $\mathbf{- 0 . 3 7 9}$ & $\mathbf{0 . 0 0 7}$ \\
PTX-3 & 0.114 & 0.425 \\
Insulin & 0.114 & 0.434 \\
WC & -0.019 & 0.893 \\
HOMA & 0.06 & 0.678
\end{tabular}

